\title{
What's Next? NCCN Gathers Healthcare Policy Experts to Deliberate Challenges to Patient Safety and Access to Cancer Care Under the New Administration
}

Patient safety has long been recognized as an integral component of high-quality and effective medical care. The stakes are especially high in oncology, where avoiding errors is imperative to delivering safe and effective radiation therapy, chemotherapy, and other high-risk treatments. Moreover, there is uncertainty surrounding shifting healthcare priorities under the Trump administration and subsequent implications for safety and access to high-quality patient-centered cancer care.

During the NCCN Policy Summit: Ensuring Patient Access and Safety in Cancer Care, experts considered these and other pertinent issues, including changing paradigms in cancer treatment, access to clinical trials and "Right to Try" legislation, and patient safety and access issues within the overall context of an increasing focus on defining and delivering high-quality cancer care through payment models based on value rather than volume. The summit took place on Thursday, June 15, 2017, at the National Press Club in Washington, DC.

"Addressing safety issues throughout the cancer care continuum must be met with an increased focus on guidelines, awareness, resources, and training," said F. Marc Stewart, MD, oncologist and Medical Director, Seattle Cancer Care Alliance, and Co-Chair of the NCCN Best Practices Committee. "Understanding patient safety issues from patient, provider, and cancer center perspectives and recognizing the innovative approaches to address these gaps are integral components of high-quality cancer care."

Clifford Goodman, PhD, of The Lewin Group moderated the summit, which consisted of short presentations followed by roundtable discussions with lively discourse.

As developers of the widely used NCCN Clinical Practice Guidelines in Oncology (NCCN Guidelines) that set the standard of cancer care in the United States, NCCN is invested in provision of high-quality, safe, value-based cancer care. Moreover, the library of NCCN Chemotherapy Order Templates (NCCN Templates) include chemotherapy, immunotherapy, supportive care agents, monitoring parameters, and safety instructions based directly on recommendations within the NCCN Guidelines. The NCCN Templates were initially published as a result of the first NCCN patient safety policy summit held in 2006, and today NCCN has published more than 1,500 chemotherapy order templates, which have been integrated into various electronic health record platforms for use at point of care.

\section{NCCN and CancerLinQ Collaborating to Provide Evidence-Based, Decision-Making Resources to Physicians}

NCCN and CancerLinQ LLC, a wholly owned nonprofit subsidiary of ASCO, recently announced a collaboration to provide a link to the NCCN Web site for easy access to the NCCN Drugs \& Biologics Compendium (NCCN Compendium) within CancerLinQ, a big data platform aimed at improving quality of care for patients with cancer. This resource will support CancerLinQ physicians and provides evidence-based guidance regarding the appropriate use of drugs and biologics in patients with cancer.

"We are pleased to collaborate with ASCO and CancerLinQ to support clinician access to evidence-based resources through technology at the point of care," said Robert W. Carlson, MD, Chief Executive Officer, NCCN. "Based directly on the NCCN Guidelines, the compendium will benefit CancerLinQ users by providing authoritative, scientifically derived information designed to support decision-making about the appropriate use of drugs and biologics in patients with cancer."

With this collaboration, CancerLinQ subscribers will now have access to a link that will bring them directly to the NCCN Compendium, where they can subscribe, for a fee. 
NCCN is able to make available its resources to the growing CancerLinQ network, which Cont. from page xxviii. includes thousands of oncologists treating millions of patients from a variety of practice types and institutions across the United States. This collaboration represents one of the ways in which NCCN is empowering physicians to access NCCN resources through everyday health information technology (HIT) workflow.

"CancerLinQ exists in service to our members to improve quality," said Kevin Fitzpatrick, Chief Executive Officer, CancerLinQ LLC. "As we continue to convene collaborators across the oncology community, we encourage efforts that enable resources to be more easily accessible for providers to make informed and timely decisions in the delivery of care to their patients. This collaboration is just one more positive step in that direction."

The NCCN Compendium contains recommendations for the appropriate use of drugs and biologics to support decision-making for patients with cancer. The recommendations are derived directly from relevant NCCN Clinical Practice Guidelines in Oncology (NCCN Guidelines), along with their clinical context, route of administration, recommended use, and NCCN category of evidence. In addition to NCCN Guidelines-specific indication and use, NCCN adds relevant information, such as pharmacologic class, relevant classification codes, and FDA indication, to the searchable database. All information is reviewed by members of the relevant NCCN Guidelines Panel before publication.

The CancerLinQ platform is a big data initiative focused on cancer patient medical information, aimed at improving the quality of patient care and outcomes. It is the only major cancer data initiative being developed and led by physicians. When complete, CancerLinQ will unlock real-world patient care data from millions of electronic health records and securely process and analyze the data to provide immediate quality feedback and clinical decision support to providers. Doctors will receive personalized insights on a scope that was previously unattainable, and patients will benefit by having access to high quality care based on up-to-date insights and findings.

The NCCN Guidelines are the recognized standard for clinical policy in cancer care and are the most thorough and most frequently updated clinical practice guidelines available in any area of medicine. For more information, visit NCCN.org.

\section{NCCN Awards Grants to Investigators at Member Institutions to Study Osimertinib in Lung Cancer}

NCCN Oncology Research Program (ORP) has funded 2 investigators from NCCN Member Institutions through a collaborative scientific research relationship with AstraZeneca to further evaluate the clinical effectiveness of osimertinib in the treatment of epidermal growth factor receptor-positive $(\mathrm{EGFRm}+)$ non-small cell lung cancer (NSCLC).

The following studies were awarded funding through NCCN ORP:

- Daniel Gomez, MD, The University of Texas MD Anderson Cancer Center: Randomized Phase II Trial of Osimertinib with or without Local Consolidation Therapy (LCT) for Patients with EGFR-mutant Metastatic NSCLC (NORTHSTAR)

- Pasi A. Jänne, MD, PhD, Dana-Farber/Brigham and Women's Cancer Center | Massachusetts General Hospital Cancer Center: A Phase 2 Study of Osimertinib in Combination with Selumetinib in EGFR Inhibitor Naïve Advanced EGFR Mutant Lung Cancer

"NCCN ORP congratulates Dr. Gomez and Dr. Jänne on their awards and looks forward to the commencement of their studies," said Susan Most, RN, MBA, Director, Clinical Operations, NCCN ORP. "We are pleased to be working with AstraZeneca on further evaluation of osimertinib, an important targeted agent for the treatment of EGFRm+ NSCLC. This project furthers NCCN's commitment to improving the lives of 
July 2017

patients with cancer by providing opportunities to identify additional treatment options Cont. from page xxxv. in lung cancer."

Submissions were peer-reviewed by the NCCN Osimertinib Scientific Review Committee. The funded concepts were selected based on several criteria, including scientific merit, existing data, and the types of studies necessary to further evaluate the efficacy of osimertinib.

NCCN ORP draws upon the expertise of investigators at the NCCN Member Institutions and their affiliates to facilitate all phases of clinical research. This research is made possible by collaborations with pharmaceutical and biotechnology companies in order to advance therapeutic options for patients with cancer. To date, this successful research model has received more than $\$ 60$ million in research grants and supported more than 140 studies, producing a number of publications in peer-reviewed journals.

To learn more about the NCCN ORP and ongoing clinical trials, visit NCCN.org/ORP. 